\title{
An examination of whether associations exist between maternal and neonatal 25OHD and infant size and adiposity at birth, 6-9 months and 2-2.5 years of age - a longitudinal observational study from the ROLO study
}

Mary K. Horan' ${ }^{1}$, Jean M. Donnelly ${ }^{1}$, Malachi J. McKenna², Brenda Crosbie ${ }^{3}$, Mark T. Kilbane ${ }^{3}$ and Fionnuala M. McAuliffe $e^{1 *}$

\begin{abstract}
Background: Vitamin D status in pregnancy and offspring bone health effects are well established, yet limited knowledge exists on the effect of maternal vitamin D status on offspring size/adiposity. This study examines the association of early (13 weeks), late (28 weeks) pregnancy and neonatal (umbilical) 25-hydroxyvitamin D (25OHD) on offspring size/adiposity.
\end{abstract}

Methods: This analysis included mother-infant pairs from the ROLO study at birth $(n=292), 6-9$ months $(n=160)$ and $2-2.5$ years $(n=287)$ postpartum.

Results: Using Institute of Medicine 2011 Report criteria, 30\% of women in early pregnancy and 38\% in late pregnancy were at risk of vitamin D deficiency $(25 \mathrm{OHD}<30 \mathrm{nmol} / \mathrm{L})$. Birthweight was negatively associated with early-pregnancy 250HD ( $p=0.004)$ and neonatal 25OHD $(p<0.001)$. Birth length was not associated with $250 H D$. Neonatal measures of overall adiposity were negatively associated with neonatal $25 \mathrm{OHD}(p=0.001$, and $p=<0.001$ respectively). At $2-2.5$ years there was a negative association between weight-for-age z-score and early-pregnancy 25OHD $(p<0.041)$.

Conclusions: Maternal and neonatal 250HD were negatively associated with offspring size/adiposity at birth and offspring weight-for-age at 2-2.5 years. Results may not reflect a general population replete in vitamin $D$, due to high prevalence of macrosomia and high risk of deficiency in this cohort. Improvement of pregnancy vitamin D status remains a public health concern.

Trial registration: Current Controlled Trials ISRCTN54392969. 22/04/2009 retrospectively registered.

Keywords: Fetal programming, Maternal vitamin D, Offspring adiposity, Childhood obesity, Maternal nutrition, ROLO study

PACS: 10101

\footnotetext{
* Correspondence: fionnuala.mcauliffe@ucd.ie

${ }^{1}$ UCD Obstetrics and Gynaecology, School of Medicine and Medical Science,

University College Dublin, National Maternity Hospital, Dublin 2, Ireland

Full list of author information is available at the end of the article
} 


\section{Background}

The role of vitamin D in bone health is well established, while evidence of its role in extra-skeletal health continues to accumulate $[1,2]$. Vitamin $D$ is derived from oral intake and from skin production by UVB radiation. Vitamin D derived from both sources is then hydroxylated in the liver to make 25-hydroxyvitamin D (25OHD) and again in the kidney to the most active form 1, 25-dihydroxyvitamin D. In Ireland the main contribution to dietary vitamin $D$ is from meat, followed to a lesser extent by fish, spreads, eggs and milk and yogurts [3]. The different sources of vitamin $\mathrm{D}$ increase the complexity of research in the area. Production due to sun exposure depends on multiple factors including latitude and UltraViolent B (UVB) radiation levels, skin colour, sunscreen use, covering up and practices such as purdah [4]. Similarly, oral intake depends on vitamin $\mathrm{D}$ levels in plant and animal foods consumed, food fortification, supplement intake, and practices such as vegetarianism and veganism $[5,6]$.

During pregnancy, maternal 25OHD tends to decline, but maternal production of 1, 25-dihydroxyvitamin D increases because there is up regulation of renal enzymes in order to facilitate the doubling of maternal intestinal calcium absorption during pregnancy [7-9]. Vitamin D is not required by the fetus for calcium absorption because calcium is actively transported across the placenta; vitamin $\mathrm{D}$ becomes essential for calcium absorption only postpartum [8]. Offspring vitamin D status at birth may be determined by measuring umbilical cord 25OHD, which correlates with maternal 25OHD [8, 10, 11]. The placenta contains 1 -hydroxylase which converts $25 \mathrm{OHD}$ to the active form but its contribution to maternal calcium balance is uncertain $[8,10,12]$.

There is evidence that maternal and fetal vitamin $D$ status or season of birth may be associated with offspring adiposity [13-17]. However, some studies have reported no such associations between maternal 25OHD and offspring health [18-23]. The aim of this study was to examine the association between maternal and neonatal 25OHD levels and infant size and adiposity at birth, 6-9 months and 2-2.5 years of age in a cohort from the ROLO (Randomised cOntrol trial of LOw glycaemic index diet versus no dietary intervention to prevent recurrence of fetal macrosomia) study [24]. In a previous analysis of a subgroup of the ROLO study, late maternal 25OHD above the cohort median was associated with significantly greater neonatal length than late maternal 25OHD below the median [25].

\section{Methods}

Mother and infant pairs from the ROLO study were included in this analysis at birth $(n=292)$, at 6-9 months postpartum $(n=160)$, and at $2-2.5$ years postpartum $(n=287)$. Of these, 159 were assessed at all three time- points (birth, 6 months and 2 years of age) and had a measurement of maternal 250HD in early pregnancy; 166 were assessed at all three time-points and had a measurement of maternal 25OHD in late pregnancy; and, 120 were assessed at all three time-points and had a measurement of neonatal 25OHD. The ROLO study was a randomised control trial of low glycaemic index diet in pregnancy to reduce macrosomia. 800 euglycaemic secundigravid women who had previously given birth to a macrosomic baby (> $4 \mathrm{~kg}$ ), and were therefore at increased risk of delivering another macrosomic infant [26], were randomised to receive low glycaemic index (GI) dietary advice or usual antenatal care, which did not include dietary advice. Detailed methodology and results of the ROLO study have been published previously $[24,27]$. In brief, the primary outcome was a reduction in birthweight and the secondary outcomes were a reduction in gestational weight gain and glucose intolerance. The primary outcome was not achieved while the secondary outcomes were both achieved. Low GI dietary advice was given at week 14 of pregnancy and demographic, well-being and lifestyle questionnaires were returned by 28 weeks gestation. 3-day food diaries were completed during each trimester of pregnancy and used to determine the glycaemic index and glycaemic load of the women's diets, both of which were significantly reduced following the intervention [24, 28]. This study was conducted according to the guidelines laid down in the Declaration of Helsinki with institutional ethics approval from ethics committee of the National Maternity Hospital, Ireland, for the original study, and further ethical approval from the same ethics committee for the followup study up to 2 years of age was obtained. Informed written maternal consent was obtained at each time-point of the study. Trial Registration with Current Controlled Trials www.controlled-trials.com ISRCTN54392969. Informed written maternal consent was obtained from all participants both during pregnancy and at the 2 year follow-up appointment.

\section{Inclusion and exclusion criteria}

Participants were secundigravida women who had previously given birth to a macrosomic infant (> $4 \mathrm{~kg}$ ). They were required to have sufficient literacy and English language fluency to understand the intervention and complete questionnaires. Participants were required to have healthy, singleton pregnancies with no intrauterine growth abnormalities. All participants were invited to return for a follow-up appointment with their infant both at 6 months postpartum and at 2 years postpartum. Infants were eligible for inclusion until they reached 9 months of age for the first postnatal follow-up and 2 years 6 months of age for the second postnatal follow-up. Participants who returned for follow-up at 2 years postpartum were chosen 
for 25OHD analysis of stored blood samples and their anthropometric measurements at birth and at 6 months were also examined, where available. It was of interest to examine long-term associations between 25OHD in pregnancy and offspring adiposity; 2 years was the latest follow-up age available so a sample of convenience was chosen.

\section{Maternal demographics, lifestyle and infant feeding practices}

Of the 800 participants of the ROLO study, 25OHD was measured in 300 participants. Of these, 292 anthropometric measurements were performed for 292 infants at birth, 160 at $6-9$ months of age, and 287 at $2-2.5$ years of age. Questions on lifestyle habits were taken from SLAN (Survey of Lifestyle, Attitudes and Nutrition in Ireland) including questions on physical activity, smoking, education and food label reading [29].

\section{Maternal and infant anthropometry}

Maternal weight, height and mid-upper arm circumference were measured at the first antenatal consultation and body mass index was calculated. Maternal weight and mid-upper arm circumference were also measured at 6-9 months and 2-2.5 years postpartum and body mass index (BMI) calculated. Neonatal weight and length was measured for all infants at birth while mid-upper arm, abdominal, hip and thigh circumference, and biceps, triceps, subscapular and thigh skinfold thickness measurements were initiated later in the study and therefore complete anthropometric data was available for only 122 neonates in this cohort. Birthweight centile was calculated using Gestation Network's Bulk Calculator version 6.2.3, UK. Centiles were corrected for maternal weight, height, parity and ethnicity and infant gestational age at delivery and gender [30]. The same anthropometric measurements were also taken at 6-9 months and 2-2.5 years of age. WHO growth standards were used to convert these measurements to z-scores which adjusts for infant age at exam (in days) and gender and report standard deviations away from the mean [31, 32]. Six-nine month and 2-2.5 year olds were also classified according to WHO BMI-for-age z-score cut-offs as wasted or severely wasted (BMI-for-age z-score $<-2$ ), normal (BMI-for-age $z$ score -2 to 1 ), at risk of overweight (BMI-for-age $\mathrm{z}$-score 1.01-2), overweight (BMI-for-age z-score 2.01-3) and obese (BMI-for age $\mathrm{z}$-score $>3$ ). Waist:hip, waist:length and subscapular skinfold:triceps skinfold ratios as well as sum of triceps and subscapular skinfold thicknesses and sum of all skinfold thicknesses were calculated as markers of infant adiposity.

Maternal supplement use, season of pregnancy, ethnicity and infant feeding practices

Information on maternal supplement use was collected during pregnancy as a binary yes/no question. Specific data on maternal vitamin D supplementation or sunlight exposure were not collected. The season of pregnancy was classified into summer or winter according to the month in which the baby was delivered; delivery from June to November was classified as a summer pregnancy while delivery from December to May was classified as a winter pregnancy [25]. Maternal ethnicity was recorded. At 6-9 months and 2-2.5 years postpartum mothers completed a questionnaire about feeding practices, such as whether or not infant had ever been breastfed, the duration of breastfeeding, the age at which solids and other drinks, including infant formula, were introduced.

\section{HD measurement}

Maternal blood samples were taken in early pregnancy at the first antenatal appointment at 13 weeks gestation and in late pregnancy at 28 weeks gestation. Cord blood samples were taken at delivery. These samples were centrifuged at $3000 \mathrm{rpm}$ for $10 \mathrm{~min}$ and serum subaliquoted and stored at minus $80{ }^{\circ} \mathrm{C}$ until analysis. Samples were excluded from analysis if they were haemolysed (early pregnancy $n=0$, late pregnancy $n=2$, cord $n=27$ ). Serum total 25OHD concentrations were measured using the Elecsys vitamin D total automated competitive binding protein assay (Roche Diagnostics $\mathrm{GmbH}$, Mannheim, Germany). This method employs a recombinant vitamin $\mathrm{D}$ binding protein as capture protein and is standardised against liquid chromatography tandem mass spectroscopy, which in turn has been standardised to The National Institute for Standards and Technology standard. Our laboratory has a proficiency certificate issued consecutively over the last 10 years, acknowledging the fact that our assay meets the performance standards set by the world's leading vitamin D quality assurance scheme (DEQAS). The Roche 25OHD method does not discriminate the 3-epimer of 25OHD. Current evidence suggests that the 3-epimer of 25OHD is present in all human serum and not just neonates less than 1 year. Concentrations of 3-epimer of 25OHD accounted for $11 \%$ of maternal, $14 \%$ of cord and $25 \%$ of infant 25OHD in the paper by Bailey et al. [33] (below). One could argue therefore that 3-epimer of 25OHD levels in mother and cord blood samples are not significantly different from each other, in terms of affecting data analysis.

The coefficients of variation (CV) for the 25OHD assay determined at assay validation were: interassay $\mathrm{CV}=8.9 \%$ at a concentration of $49.5 \mathrm{nmol} / \mathrm{L}, 3.7 \%$ at $103 \mathrm{nmol} / \mathrm{L}$; intra-assay CV $=2.9 \%$ at a concentration of $49.5 \mathrm{nmol} / \mathrm{L}, 1.4 \%$ at $103 \mathrm{nmol} / \mathrm{L}$. We participate in the Vitamin D External Quality Assessment Scheme [34]. We interpreted maternal 25OHD according to North American Institute of Medicine (IOM) Dietary Reference Intake (DRI) committee, which specified a 25OHD 
corresponding to risk of deficiency at $<30 \mathrm{nmol} / \mathrm{L}$, to the estimated average requirement (EAR) at $40 \mathrm{nmol} / \mathrm{L}$, and to the recommended daily allowance at $50 \mathrm{nmol} / \mathrm{L}[2,35]$.

\section{Statistical analysis}

The study represents a sub-analysis of a primary randomized control trial, ROLO, which was designed with very different hypotheses and objectives to those that form the basis of this report. This study was not planned a priori and represents a sample of convenience for a sub-analysis. Results are presented as mean (standard deviation) or as number (percent). Statistical analyses entailed simple and multiple linear regression modelling using a forced enter approach. Known confounders were controlled for in these models as follows: (1) at birth, these variables were season of pregnancy, maternal education, maternal BMI in early pregnancy, any supplement use during pregnancy, maternal smoking, offspring gender and gestational age; and (2) at 6-9 months and 2-2.5 years, these variables were season of pregnancy, maternal education, any supplement use during pregnancy, maternal smoking, offspring gender and age at assessment (weeks) and finally duration of breastfeeding. Models predictive of infant anthropometric measurements, for which z-scores were available, were not adjusted for infant age and gender as these factors are taken into account when converting to z-scores. Control and intervention groups were analysed together but study group was controlled for in all final models. Multiple linear regression resulted in a best and final model, and models that were statistically significant $(p<0.05)$ were reported. For 6 month and 2 year olds, they were also grouped into the dichotomies "not overweight or at risk of overweight" and "overweight or obese" using World Health Organisation (WHO) BMI-for age cut-off [32]. Due to small numbers in the wasted and overweight and obese groups; the association of maternal and neonatal 25OHD with these dichotomies was then further analysed using t-tests. Statistical analysis was performed using IBM SPSS for Windows, version 20.0 (Armonk, NY).

\section{Results}

\section{Background demographics}

Maternal demographics and offspring anthropometry are displayed in Table 1. There was no difference in background demographics between the control and intervention groups similar to the original ROLO study [24]. Mothers in this cohort were relatively well educated with $56.1 \%$ having completed third level education. Maternal age at delivery was $33.0 \pm 3.9$ years. Maternal BMI was $26.07 \pm 4.40 \mathrm{~kg} / \mathrm{m}^{2}$ and $1.7 \%$ of mothers reported smoking during pregnancy. Although the intervention group of this cohort had significantly lower post-intervention
Table 1 Background characteristics of the ROLO cohort for whom vitamin D levels were measured

\begin{tabular}{|c|c|}
\hline Variable & Mean \\
\hline Early pregnancy $25 \mathrm{OHD}(\mathrm{nmol} / \mathrm{L})$ & 41.8 \\
\hline Late pregnancy 25OHD (nmol/L) & 40.0 \\
\hline Neonatal vitamin $25 \mathrm{OHD}(\mathrm{nmol} / \mathrm{L})$ & 44.6 \\
\hline Mother age at delivery (years) & 33.0 \\
\hline Mother height (cm) & 165 \\
\hline Mother weight booking (kg) & 72.1 \\
\hline Mother MUAC at booking $(\mathrm{cm})$ & 29.2 \\
\hline Mother BMI at booking $\left(\mathrm{kg} / \mathrm{m}^{2}\right)$ & 26.1 \\
\hline Gestational weight gain (kg) & 13.6 \\
\hline Length of gestation (days) & 283 \\
\hline Birthweight (kg) & 4.06 \\
\hline Birthweight centile & 72.5 \\
\hline Birth length $(\mathrm{cm})$ & 52.9 \\
\hline Birth head circumference $(\mathrm{cm})$ & 35.8 \\
\hline Birth abdominal circumference $(\mathrm{cm})$ & 33.6 \\
\hline Birth chest circumference (cm) & 35.6 \\
\hline Birth hip circumference (cm) & 33.7 \\
\hline Birth mid upper arm circumference $(\mathrm{cm})$ & 12.6 \\
\hline Birth thigh circumference $(\mathrm{cm})$ & 16.2 \\
\hline Birth subscapular SFT (mm) & 6.95 \\
\hline Birth biceps SFT (mm) & 6.72 \\
\hline Birth triceps SFT (mm) & 6.97 \\
\hline Birth thigh SFT (mm) & 7.81 \\
\hline Birth waist:hip circumference ratio & 1.00 \\
\hline Birth waist circumference:length ratio & 0.64 \\
\hline Birth sum of all SFT (mm) & 28.5 \\
\hline Birth sum of triceps SFT and subscapular SFT (mm) & 13.9 \\
\hline Birth subscapular SFT:triceps SFT ratio & 1.02 \\
\hline Age at 6-9 month assessment (weeks) & 28.2 \\
\hline 6-9 month weight-for-age z-score & 0.74 \\
\hline 6-9 month length-for-age z-score & 1.30 \\
\hline 6-9 month BMI-for-age z-score & 0.02 \\
\hline Age at 2-2.5 year assessment (weeks) & 109 \\
\hline 2-2.5 year weight-for-age $z$-score & 0.69 \\
\hline $2-2.5$ year length-for-age $z$-score & 0.90 \\
\hline 2-2.5 year BMl-for-age $z$-score & 0.40 \\
\hline Duration of Breastfeeding (weeks) & 13.2 \\
\hline
\end{tabular}

SFT skinfold thickness, MUAC mid-upper arm circumference

glycaemic index in trimester 2 and 3 similarly to the original ROLO study $(56.4 \pm 3.9$ vs. $57.7 \pm 3.4, p=0.010$ and $55.7 \pm 3.8$ vs. $57.7 \pm 3.9, p<0.001$ respectively) there was no significant difference in gestational weight gain between the two groups $(p=0.570)$ unlike in the original study [24]. According to the World Health 
Organisation (WHO) BMI-for-age classification [32] at 6-9 months, 7 infants (4.4\%) were classified as wasted, $133(83.1 \%)$ as normal, $14(8.8 \%)$ as at risk of overweight, $6(3.8 \%)$, and as overweight or obese (0\%). At $2-2.5$ years of age, according to the WHO BMI-for-age classification, 2 infants $(0.7 \%)$ were classified as wasted, 207 (71.9\%) as normal, 51 (17.7\%) as at risk of overweight, 25 (8.7\%) as overweight and $3(1.0 \%)$ as obese. Mothers who participated at 2-2.5 years were compared with those who did not and were found to have a higher education level, were less likely to be smokers and had a lower BMI at baseline than those who did not attend (Additional file 1: Table S1). These factors were controlled for in the analysis.

\section{Maternal supplement use, season of pregnancy, ethnicity and infant feeding practices}

Maternal ethnicity was mainly "white Irish" (92.8\%) with only $0.3 \%$ of women classified as Filipino or South East Asian, $0.3 \%$ as Indian and $0.3 \%$ as Chinese, while $6.2 \%$ were "white other". The majority of women (56.6\%) were taking some vitamin or mineral supplement during pregnancy, although type of supplement was not recorded. The season of pregnancy was fairly evenly spread as $55.1 \%$ of women were classified as having winter pregnancies and $44.9 \%$ summer pregnancies. $46.2 \%$ of women reported ever breastfeeding the participant infant, while $26.4 \%$ reported never breastfeeding and $27.4 \%$ did not provide data on breastfeeding. The duration of breastfeeding in the overall cohort was $13.2 \pm 23.6$ weeks although the duration of breastfeeding for women who reported ever breastfeeding was higher at $28.5 \pm 27.7$ weeks. Again, there was no difference between the control and intervention groups for any of these variables.

\section{HD measurements}

There was no difference in 25OHD between the control and intervention groups in early or late pregnancy or neonatal blood samples $(p=0.568, p=0.074$ and $p=0.264$ respectively). Mean (SD) 25OHD was 41.8 (19.5) $\mathrm{nmol} / \mathrm{L}$ in early pregnancy, 39.9 (21.4) $\mathrm{nmol} / \mathrm{L}$ in late pregnancy, and $44.6(26.2) \mathrm{nmol} / \mathrm{L}$ in neonatal cord blood. 25OHD in early and late pregnancy were both positively correlated with neonatal 25OHD $(r=0.676$, $p<0.001$ and $r=0.761, p<0.001$ respectively). When 25OHD concentrations were classified according to IOM guidelines [2], $30.2 \%$ of women were $<30 \mathrm{nmol} / \mathrm{L}$, $39.5 \%$ were between 30 and $50 \mathrm{nmol} / \mathrm{L}$, and $30.2 \%$ were $>50 \mathrm{nmol} / \mathrm{L}$ in early pregnancy. Of those with $25 \mathrm{OHD}<30 \mathrm{nmol} / \mathrm{L}$ in early pregnancy, $47.8 \%$ were members of the intervention group. The corresponding frequencies of women in each $25 \mathrm{OHD}$ category in late pregnancy were $37.7 \%, 34.2 \%$ and $28.1 \%$ respectively, and of those with $25 \mathrm{OHD}<30 \mathrm{nmol} / \mathrm{L}, 46.4 \%$ were members of the intervention group. There was no association between maternal BMI in early pregnancy and 25OHD in early $(p=0.271)$ or late $(p=0.137)$ pregnancy nor was there any association with early or late pregnancy $25 \mathrm{OHD}$ when the WHO BMI classification was used to categorise maternal BMI ( $p=0.811, p=0.456$ respectively). Finally, there was no association observed between vitamin D categories in early or late pregnancy and baseline or 28 weeks gestation maternal glucose levels, nor with development of gestational diabetes. Summer pregnancies (baby delivered between June and November) were associated with significantly higher $25 \mathrm{OHD}$ than winter pregnancies in late gestation $(44.1 \pm 23.8$ vs. $36.6 \pm 18.7 \mathrm{nmol} / \mathrm{L}$, $p=0.003)$ and in neonatal samples (56.0 \pm 30.7 vs. $38.2 \pm 19.7 \mathrm{nmol} / \mathrm{L}, p<0.001)$, but the opposite was seen in early pregnancy $(38.3 \pm 19.8$ vs. $46.5 \pm 18.3 \mathrm{nmol} / \mathrm{L}$, $p=0.001)$. Supplement intake was not associated with absolute levels of maternal or neonatal 25OHD in the overall cohort. However, when the cohort was separated by season, supplement intake was associated with significantly higher $25 \mathrm{OHD}$ in late pregnancy during winter $(40.0 \pm 21.1$ vs. $33.0 \pm 14.0 \mathrm{nmol} / \mathrm{L}, p=0.025)$.

\section{Association between early and late maternal 250HD and neonatal 250HD and offspring size and adiposity Birth anthropometry}

Single linear regression analysis showed many significant associations between neonatal anthropometry and early and late pregnancy and neonatal 25OHD (Table 2), but after adjustment for many confounders in the multiple linear regression models there were fewer significant associations (Table 3). After adjustment for confounders, birthweight was negatively associated with early pregnancy and neonatal 25OHD $(\beta=-4.158, p=0.004$ and $\beta=-5.919, P<0.001$, respectively). Birth length was also negatively associated with early, late pregnancy 25OHD and with neonatal 25OHD; but when birthweight was controlled for in these models, this association lost significance at all time-points $(p=0.123, p=0.117$ and $p=0.244$, respectively). Neonatal 25OHD was negatively associated with the following at birth: subscapular skinfold thickness $(\beta=-0.024, p<0.001)$, sum of all skinfold thicknesses, a measure of overall adiposity, $(\beta=-0.079$, $p=0.001)$, and with the sum of the triceps and subscapular skinfold thickness $(\beta=-0.043, p<0.001)$. Ponderal index at birth was not associated with early or late pregnancy or neonatal $25 \mathrm{OHD}$.

\section{Six-nine month anthropometry}

There were no statistically significant associations observed at 6-9 months of age when confounders were controlled for using multiple linear regression. BMI-for-age dichotomies "not overweight or at risk of overweight" vs. "at risk of overweight or overweight or obese" were not 
Table 2 Simple linear regression showing associations between 25OHD (independent variable) and offspring anthropometrics (dependent variables)

\begin{tabular}{|c|c|c|c|c|c|}
\hline Variable & $\beta$ & SE & $P$ & $\mathrm{~F}$ & $\mathrm{R}^{2}$ \\
\hline \multicolumn{6}{|l|}{ Early pregnancy $25 \mathrm{OHD}(\mathrm{nmol} / \mathrm{L})$} \\
\hline Birthweight (g) & -3.85 & 1.37 & 0.005 & 7.88 & 0.023 \\
\hline Birthweight centile & -0.25 & 0.08 & 0.001 & 11.3 & 0.036 \\
\hline Birth length (cm) & -0.01 & 0.01 & 0.001 & 2.72 & 0.007 \\
\hline Birth head circumference (cm) & -0.01 & 0.01 & 0.013 & 6.28 & 0.037 \\
\hline 6 month head circumference for age $z$-score & -0.01 & 0.01 & 0.021 & 5.44 & 0.028 \\
\hline 2 year weight for age $z$ score & -0.01 & 0.00 & 0.019 & 5.54 & 0.016 \\
\hline \multicolumn{6}{|l|}{ Late pregnancy $25 \mathrm{OHD}$ (nmol/L) } \\
\hline Birthweight (g) & -1.26 & 1.27 & 0.321 & 0.99 & 0.000 \\
\hline Birthweight centile & -0.11 & 0.07 & 0.126 & 2.36 & 0.005 \\
\hline Birth length (cm) & -0.01 & 0.01 & 0.057 & 3.66 & 0.011 \\
\hline 6 month subscapular SFT:triceps SFT ratio & -0.002 & 0.00 & 0.040 & 4.27 & 0.022 \\
\hline 2 year waist:hip ratio & -0.001 & 0.00 & 0.007 & 7.52 & 0.027 \\
\hline \multicolumn{6}{|l|}{ Neonatal 25OHD (nmol/L) } \\
\hline Birthweight (g) & -4.46 & 1.18 & $<0.001$ & 14.2 & 0.054 \\
\hline Birthweight centile & -0.24 & 0.06 & $<0.001$ & 15.2 & 0.060 \\
\hline Birth length (cm) & -0.02 & 0.01 & 0.020 & 5.52 & 0.023 \\
\hline Birth abdominal circumference $(\mathrm{cm})$ & -0.02 & 0.01 & 0.016 & 6.05 & 0.044 \\
\hline Birth thigh circumference $(\mathrm{cm})$ & -0.01 & 0.01 & 0.029 & 4.92 & 0.035 \\
\hline Birth subscapular SFT (mm) & -0.02 & 0.01 & $<0.001$ & 13.7 & 0.123 \\
\hline Birth biceps SFT (mm) & -0.01 & 0.01 & 0.033 & 4.68 & 0.039 \\
\hline Birth sum of subscapular and triceps SFT (mm) & -0.03 & 0.01 & 0.002 & 9.97 & 0.091 \\
\hline Birth subscapular SFT:triceps SFT ratio & -0.002 & 0.00 & 0.050 & 3.94 & 0.032 \\
\hline Birth sum of SFT (mm) & -0.06 & 0.02 & 0.005 & 8.35 & 0.076 \\
\hline 6 month waist:length ratio & 0.00 & 0.00 & 0.014 & 6.19 & 0.041 \\
\hline
\end{tabular}

SFT skinfold thickness, $R^{2}$ adjusted coefficient of determination

associated with early or late pregnancy 25OHD ( $p=0.170$ and $p=0.937$, respectively) or with neonatal 25OHD $(p=0.463)$.

\section{Two-two and a half year anthropometry}

The only significant association remaining at $2-2.5$ years once confounders were controlled for using multiple linear regression was a negative association between early pregnancy $25 \mathrm{OHD}$ and 2-2.5 year old weight-for-age z-score $(\beta=-0.11, p<0.001)$ (Table 3). BMI-for-age dichotomies "not overweight or at risk of overweight" versus "at risk of overweight or overweight or obese" were not associated with early or late pregnancy 250HD ( $p=0.250$ and $p=0.599$ respectively) or with neonatal 25OHD $(p=0.326)$.

\section{Discussion}

The average 25OHD in our maternal cohort, both in early and late gestation, is nearly equivalent to the IOM EAR for healthy population at $40 \mathrm{nmol} / \mathrm{L}[2,36]$ but $30 \%$ in early pregnancy and $37 \%$ in late pregnancy were at risk of vitamin D deficiency $(25 \mathrm{OHD}<30 \mathrm{nmol} / \mathrm{L})$. These estimates of risk of vitamin D deficiency are substantially higher than prevalence estimate of $7 \%$ in healthy Irish adults in the National Adult Nutrition Survey [6]. While we have noted a marked improvement in vitamin D status in our studies over the past three decades, there are still at-risk groups in Ireland such as pregnant women especially during wintertime [25, 37-41].

We found that birthweight was negatively associated with early pregnancy and neonatal 25OHD. Birth length was negatively associated with early and late pregnancy and neonatal 25OHD; however when birthweight was controlled for in these models, this association lost significance at all time-points. Neonatal sum of all skinfolds and sum of subscapular and triceps skinfolds, both measures of overall adiposity, were negatively associated with neonatal 25OHD. There was no association between early or late pregnancy $25 \mathrm{OHD}$ or neonatal $25 \mathrm{OHD}$ and $6-$ 9 month old anthropometry. At 2-2.5 years of age the only association observed was a negative association between weight-for-age z-score and early pregnancy 25OHD. 
Table 3 Associations between early and late pregnancy and cord 25OHD and offspring size and adiposity- adjusted analysis

\begin{tabular}{|c|c|c|c|c|c|c|}
\hline Variable & B & SEB & $\mathrm{P}$ & $R^{2}$ & $\mathrm{~F}$ & $\mathrm{p}$ \\
\hline \multicolumn{7}{|l|}{ Early pregnancy $25 \mathrm{OHD}(\mathrm{nmol} / \mathrm{L})$} \\
\hline Birthweight (g) & -4.16 & 1.42 & 0.004 & 0.20 & 7.623 & $<0.001$ \\
\hline Birth length $(\mathrm{cm})$ & -0.02 & 0.01 & 0.023 & 0.20 & 6.639 & $<0.001$ \\
\hline airth length $(\mathrm{cm})$ & -0.01 & 0.01 & 0.123 & 0.29 & 9.512 & $<0.001$ \\
\hline $2-2.5$ year weight for age $z$ score & -0.11 & 0.00 & $<0.001$ & 0.12 & 4.291 & $<0.001$ \\
\hline \multicolumn{7}{|l|}{ Late pregnancy $25 \mathrm{OHD}(\mathrm{nmol} / \mathrm{L})$} \\
\hline Birthweight (g) & -2.51 & 1.34 & 0.063 & 0.18 & 6.729 & $<0.001$ \\
\hline Birth length $(\mathrm{cm})$ & -0.02 & 0.01 & 0.028 & 0.15 & 5.142 & $<0.001$ \\
\hline airth length (cm) & -0.01 & 0.01 & 0.117 & 0.29 & 9.650 & $<0.001$ \\
\hline \multicolumn{7}{|l|}{ Neonatal 25OHD (nmol/L) } \\
\hline Birthweight(g) & -5.92 & 1.20 & $<0.001$ & 0.29 & 10.198 & $<0.001$ \\
\hline Birth length $(\mathrm{cm})$ & -0.02 & 0.01 & 0.006 & 0.20 & 5.180 & $<0.001$ \\
\hline airth length $(\mathrm{cm})$ & -0.01 & 0.01 & 0.244 & 0.32 & 9.074 & $<0.001$ \\
\hline Birth subscapular SFT (mm) & -0.02 & 0.01 & $<0.001$ & 0.24 & 3.534 & 0.001 \\
\hline Birth sum of all SFT (mm) & -0.08 & 0.02 & 0.001 & 0.12 & 2.093 & 0.043 \\
\hline Birth sum of subscapular and triceps SFT (mm) & -0.04 & 0.01 & $<0.001$ & 0.16 & 2.501 & 0.016 \\
\hline
\end{tabular}

SFT skinfold thickness, $R^{2}$ adjusted coefficient of multiple determination. Multiple linear regression models for dependent variables at birth were adjusted for season of pregnancy, maternal education, maternal BMI in early pregnancy, any supplement use during pregnancy, maternal smoking,

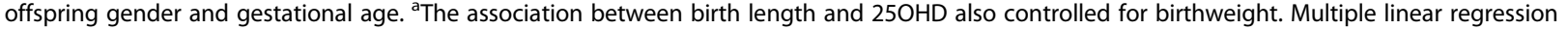
models for dependent variables at 6-9 months and 2-2.5 years were adjusted for season of pregnancy, maternal education, any supplement use during pregnancy, maternal smoking, offspring gender and age at assessment (weeks), and duration of breastfeeding

Our finding of negative associations between early pregnancy 25OHD and neonatal 25OHD and birthweight is contrary to the findings of several other studies at birth. A recent study by Eckhardt et al. found that maternal $25 \mathrm{OHD}<30 \mathrm{nmol} / \mathrm{L}$ compared to maternal $25 \mathrm{OHD} \geq 30 \mathrm{nmol} / \mathrm{L}$ at 20 weeks gestation was associated with lower weight-for-age z-score and BMI-for-age z-score at birth [17]. A study by Leffelaar et al. did not find any association between maternal 25OHD and birthweight once confounders were controlled for, but did find that maternal $25 \mathrm{OHD}<30 \mathrm{nmol} / \mathrm{L}$ compared to $25 \mathrm{OHD}>50 \mathrm{nmol} / \mathrm{L}$ was associated with greater risk of small for gestational age [42]. While another study by Morley et al. found no association between birthweight and early or late pregnancy 25OHD [43]. An older study by Phillips and Young similarly found that birthweight was not associated with month or season of birth although 25OHD was not measured [16]. The contrary nature of our findings of a negative association between maternal 25OHD in early pregnancy and offspring birthweight to those of other studies may be due, in part, to the nature of our cohort, in which there was a high prevalence of macrosomia. Our finding that late pregnancy 25OHD was not significantly associated with birthweight is similar to the study by Gale et al. of a cohort from Southampton which found no association between maternal 25OHD in late pregnancy and birthweight, and to another study by Sayers and Tobias which found that maternal UVB exposure in late pregnancy was not significantly associated with birthweight $[23,44]$.

Birth length was negatively associated with early and late pregnancy 25OHD and neonatal 25OHD; however, when birthweight was controlled for in these models, to determine if this association was simply a function of birthweight, this association lost significance at all timepoints. This finding is similar to a study by Gale et al. found no association between late pregnancy 25OHD and birth length [23]. A study by Morales et al. of early pregnancy 25OHD likewise found no association [15]. Previous studies have also reported positive associations between birth length and maternal 25OHD levels in pregnancy [17] and with maternal UVB exposure [43, 44]. Fewer studies have examined the longer-term associations of maternal or neonatal 25OHD and offspring length. In this cohort, no association was observed between neonatal $25 \mathrm{OHD}$ or maternal $25 \mathrm{OHD}$ in early or late pregnancy and offspring length at 6-9 months or 2-2.5 years of age which is similar to the results of other longitudinal studies examining these associations at various ages in childhood [15, 23, 45]. Leffelaar et al. [42] also examined offspring height, up to 1 year of age and found that infants of mothers who were vitamin $\mathrm{D}$ deficient in early pregnancy were significantly shorter than infants of mothers who were not deficient at 1 month of age. Whereas by 1 year of age this relationship had reversed and the length of infants whose mothers were vitamin D 
deficient was significantly greater than those of mother without deficiency.

Neonatal sum of all skinfolds and sum of subscapular and triceps skinfolds, both measures of overall adiposity, were negatively associated with neonatal 25OHD in this cohort contrary to results of a recent study by Godang et al. where neonatal $25 \mathrm{OHD}$ was positively associated with neonatal fat mass measured by dual electron $\mathrm{x}$-ray absorptiometry (DXA) [14]. Another study, also using DXA, found that late pregnancy $25 \mathrm{OHD}$ was positively associated with 3 week old fat mass, which the authors classified as birth adiposity, although neonatal 25OHD was not examined [13]. Finally, a study by Eckhardt et al. found that BMI-for-age z-score at birth was positively associated with maternal 25OHD at 20 weeks gestation [17].

In terms of offspring adiposity in later childhood, at 22.5 years old a negative association between weight-forage $\mathrm{z}$-score and early pregnancy $25 \mathrm{OHD}$ was observed in this cohort. Other studies that have examined the association between maternal or neonatal 25OHD and offspring adiposity in later life have reported conflicting results. Gale et al. found no association between late pregnancy 25OHD and offspring fat mass measured using DXA at 9 years of age [23]. While Crozier et al. found that late pregnancy 25OHD was negatively associated with offspring fat mass, also measured using DXA, at 6 years but not at 4 years of age [13]. Leffelaar et al. observed significantly greater offspring weight at 6 and 9 months of age in children whose mothers were deficient rather than sufficient in vitamin D in early pregnancy, however offspring adiposity was not examined [42]. A study of early pregnancy found that $25 \mathrm{OHD}<50 \mathrm{nmol} / \mathrm{L}$ was associated with increased BMI-for-age z-score and risk of overweight at 1 year of age but not at 4 years of age [15]. Whereas, Eckhardt et al. found that BMI-for-age z-score and weightfor-age $z$-score at 4 months and 1 year of age were not significantly associated with maternal vitamin D deficiency at 20 weeks gestation [17]. A study of 9.9 year old offspring found that maternal UVB exposure in late pregnancy was positively associated with offspring fat mass and weight [44]. An earlier study found that obesity at age 59-73 years was positively associated with winter pregnancies in men with a trend towards the same association in women [16].

This study had some limitations. Detailed data on maternal vitamin D supplement use and maternal sun exposure was not available, but we had data on season of pregnancy and actual measured 25OHD. In addition, as mentioned, complete anthropometric measurements were not available for all neonates although we had weight, length and head circumference for the full cohort which allowed examination of the association between maternal 25OHD and basic measures of neonatal size for the full cohort, with more in depth body composition analysis possible in a subgroup. Finally, data was not available on offspring diet at 2-2.5 years, precluding adjustment for the effect of dietary intake on size and adiposity. However, we did have data on infant feeding practices and duration of breastfeeding, which was controlled for in all analyses. Some variables controlled for in the multiple regression models may have been on the causal pathway such as: season, supplement intake or maternal BMI. However, these variables equally may have been confounders in their own right: season may affect birthweight independently of sunlight exposure [46]; supplement intake may have affected birthweight through supplementation with micronutrients other than vitamin D; and, maternal adiposity may lead to low 25OHD leading to altered birthweight. Regards the latter point, we did not find an association between maternal BMI and vitamin D status. We deemed it appropriate to control for any possible confounders while recognising the limitations of this strategy. There is no uniformity about the definition of season with respect to analysis of 25OHD. Despite improvement in vitamin D in Ireland over the past 40 years, a marked seasonality in 25OHD is still observed [47]. We chose summer/winter definition in order to align with our previous ROLO study analysis [25]. The results of our study may not be generalisable other cohorts due to relatively homogenous ethnicity, high education level and high prevalence of macrosomia and these factors, particularly the risk of macrosomia, which may explain the contradictory results when compared with other studies of maternal 25OHD and offspring size. Unfortunately, selection bias is an issue with all longitudinal studies due to loss to follow-up over time and it was an issue in this study too with mothers who returned at 2-2.5 years post-partum having a higher level of education, lower BMI in early pregnancy and lower smoking rate. However, these factors were controlled for where possible and, while further research is necessary, this study adds important data to the sparse literature on the association between maternal and neonatal $25 \mathrm{OHD}$ and offspring size and adiposity at birth and in childhood, and is unique in examining this association using early and late pregnancy and neonatal 25OHD.5.

\section{Conclusions}

Maternal and neonatal 25OHD were negatively associated with offspring size and adiposity at birth, and with offspring weight-for-age at $2-2.5$ years in a cohort with high prevalence of macrosomia in whom risk of vitamin $D$ deficiency was high. No association was observed with offspring anthropometry at 6-9 months of age. Further research is necessary to determine whether an optimum 25OHD level exists in pregnancy for promotion of healthy offspring size and adiposity. Meanwhile, improvement of vitamin $\mathrm{D}$ status in pregnancy remains a public health concern. 


\section{Additional file}

Additional file 1: Table S1. Comparison of participants at 2-2.5 years post-partum (who also had vitamin D levels from pregnancy available) and non-responders. (DOCX $15 \mathrm{~kb}$ )

\section{Abbreviations}

25OHD: 25-Hydroxyvitamin D; Gl: Glycaemic index; UV: Ultraviolet; WHO: World Health Organisation

\section{Acknowledgments}

The authors would like to thank Jacinta Byrne, Ciara McGowan and Jennifer Walsh for data collection. This study was supported by the Health Research Board Ireland, the Health Research Centre for Health and Diet Research, The National Maternity Hospital Medical Fund and the European Union's Seventh Framework Programme (FP7/2007-2013), project EarlyNutrition under grant agreement no. 289346.

\section{Funding}

This study was supported by the Health Research Board Ireland, the Health Research Centre for Health and Diet Research, The National Maternity Hospital Medical Fund and the European Union's Seventh Framework Programme (FP7/2007-2013), project EarlyNutrition under grant agreement no. 289346.

\section{Availability of data and materials}

Data will be available upon request to the authors.

\section{Authors' contributions}

FMMCA conceived of and designed the study. MH, JMD, MJMCK, MTK and $\mathrm{BC}$ were involved in data collection, analysis, and interpretation. $\mathrm{MH}$ drafted the manuscript and JMD, MJMCK, MTK and FMMcA revised it critically for important intellectual content. All authors read and approved the final manuscript

\section{Ethics approval and consent to participate}

All procedures performed in studies involving human participants were in accordance with the ethical standards of the institutional research committee and with the 1964 Helsinki declaration and its later amendments.

\section{Consent for publication}

Not applicable.

\section{Competing interests}

The authors declare that they have no competing interests.

\section{Publisher's Note}

Springer Nature remains neutral with regard to jurisdictional claims in published maps and institutional affiliations.

\section{Author details}

'UCD Obstetrics and Gynaecology, School of Medicine and Medical Science, University College Dublin, National Maternity Hospital, Dublin 2, Ireland. 'Department of Endocrinology, St. Vincent's University Hospital, Dublin 4, Ireland. ${ }^{3}$ Metabolism Laboratory, St Vincent's University Hospital, Dublin 4, Ireland.

Received: 17 January 2017 Accepted: 9 July 2017

Published online: 17 July 2017

\section{References}

1. Ross AC, Taylor CL, Yaktine AL, Del Valle HB. Dietary reference intakes for calcium and vitamin D. National Academies Press; 2011.

2. Ross AC, Manson JE, Abrams SA, Aloia JF, Brannon PM, Clinton SK, et al. The 2011 report on dietary reference intakes for calcium and vitamin D from the Institute of Medicine: what clinicians need to know. J Clin Endocrinol Metab. 2011;96:53-8

3. Irish Universities Nutrition Alliance. National Adult Nutrition Survey Summary Report. Irish University Nutrition Alliance; 2011. http://www.iuna. net/wp-content/uploads/2010/12/National-Adult-Nutrition-Survey-SummaryReport-March-2011.pdf. Accessed 15 Mar 2015
4. Hagenau T, Vest R, Gissel TN, Poulsen CS, Erlandsen M, Mosekilde L, et al. Global vitamin D levels in relation to age, gender, skin pigmentation and latitude: an ecologic meta-regression analysis. Osteoporos Int. 2009;20:13340 .

5. Black LJ, Seamans KM, Cashman KD, Kiely M. An updated systematic review and meta-analysis of the efficacy of vitamin D food fortification. J Nutr. 2012;142:1102-8.

6. Cashman KD, Muldowney S, McNulty B, Nugent A, FitzGerald AP, Kiely M, et al. Vitamin $D$ status of irish adults: findings from the national adult nutrition survey. Br J Nutr. 2012;25:1248-56.

7. Food Safety Authority of Ireland. Recommended Dietary Allowances for Ireland: Nutrition. Food Safety Authority of Ireland; 1999. https://www.fsai.ie/ assets/0/86/204/fb3f2891-2896-4bf9-903f-938f3c2ad01f.pdf. Accessed 15 Mar 2015

8. Kovacs CS. Vitamin D in pregnancy and lactation: maternal, fetal, and neonatal outcomes from human and animal studies. Am J Clin Nutr. 2008;88:520S-8S.

9. Hollis BW, Johnson D, Hulsey TC, Ebeling M, Wagner CL. Vitamin D supplementation during pregnancy: double-blind, randomized clinical trial of safety and effectiveness. J Bone Miner Res. 2011;26:2341-57.

10. Lucas RM, Ponsonby A-L, Pasco JA, Morley R. Future health implications of prenatal and early-life vitamin D status. Nutr Rev. 2008;66:710-20.

11. Viljakainen HT, Saarnio $E$, Hytinantti T, Miettinen $M$, Surcel $H$, Mäkitie $O$, et al. Maternal vitamin D status determines bone variables in the newborn. J Clin Endocrinol Metab. 2010;95:1749-57.

12. Hollis BW, Wagner CL. Vitamin D and pregnancy: skeletal effects, nonskeletal effects, and birth outcomes. Calcif Tissue Int. 2013;92:128-39.

13. Crozier SR, Harvey NC, Inskip HM, Godfrey KM, Cooper C, Robinson SM. Maternal vitamin D status in pregnancy is associated with adiposity in the offspring: findings from the Southampton Women's Survey. Am J Clin Nutr. 2012;96:57-63.

14. Godang K, Frøslie KF, Henriksen T, Qvigstad E, Bollerslev J. Seasonal variation in maternal and umbilical cord $25(\mathrm{OH})$ vitamin D and their associations with neonatal adiposity. Eur J Endocrinol. 2014;170:609-17.

15. Morales E, Rodriguez A, Valvi D, Iñiguez C, Esplugues A, Vioque J, et al. Deficit of vitamin $D$ in pregnancy and growth and overweight in the offspring. Int J Obes. 2015:39:61-8.

16. Phillips DI, Young JB. Birth weight, climate at birth and the risk of obesity in adult life. Int J Obes Relat Metab Disord. 2000;24:281-7.

17. Eckhardt CL, Gernand AD, Roth DE, Bodnar LM. Maternal vitamin D status and infant anthropometry in a US multi-centre cohort study. Ann Hum Biol. 2014:42:217-24

18. Marjamäki L, Niinistö S, Kenward MG, Uusitalo L, Uusitalo U, Ovaskainen ML et al. Maternal intake of vitamin D during pregnancy and risk of advanced beta cell autoimmunity and type 1 diabetes in offspring. Diabetologia. 2010:53:1599-607.

19. Miettinen ME, Reinert $L$, Kinnunen $L$, Harjutsalo $V$, Koskela $P$, Surcel H-M, et al. Serum 25-hydroxyvitamin D level during early pregnancy and type 1 diabetes risk in the offspring. Diabetologia. 2012;55:1291-4.

20. Williams DM, Fraser A, Fraser WD, Hypponen E, Smith GD, Deanfield J, et al Associations of maternal 25-hydroxyvitamin D in pregnancy with offspring cardiovascular risk factors in childhood and adolescence: findings from the avon longitudinal study of parents and children. Heart. 2013;99:1849-56.

21. Tornhammar P, Ueda P, Hult M, Simila H, Eyles D, Norman M. Season of birth, neonatal vitamin D status, and cardiovascular disease risk at 35 y of age: a cohort study from Sweden. Am J Clin Nutr. 2014:99:472-8.

22. Pike KC, Inskip HM, Robinson S, Lucas JS, Cooper C, Harvey NC, et al. Maternal late-pregnancy serum 25 -hydroxyvitamin $\mathrm{D}$ in relation to childhood wheeze and atopic outcomes. Thorax. 2012; doi:10.1136/ thoraxjnl-2012-201888

23. Gale CR, Robinson SM, Harvey NC, Javaid MK, Jiang B, Martyn CN, et al. Maternal vitamin D status during pregnancy and child outcomes. Eur J Clin Nutr. 2008:62:68-77.

24. Walsh JM, McGowan CA, Mahony R, Foley ME, McAuliffe FM. Low glycaemic index diet in pregnancy to prevent macrosomia (ROLO study): randomised control trial. BMJ. 2012;345:e5605

25. Walsh JM, Kilbane M, McGowan CA, McKenna MJ, McAuliffe FM. Pregnancy in dark winters: implications for fetal bone growth? Fertil Steril. 2013. doi:10.1016/j.fertnstert2012.09.010.

26. Mahony R, Walsh C, Foley ME, Daly L, O'Herlihy C. Outcome of second delivery after prior macrosomic infant in women with normal glucose tolerance. Obstet Gynecol. 2006;107:857-62. 
27. Walsh J, Mahony R, Foley M, Mc AF. A randomised control trial of low glycaemic index carbohydrate diet versus no dietary intervention in the prevention of recurrence of macrosomia. BMC Pregnancy Childbirth. 2010;10:16.

28. McGowan CA, Walsh JM, Byrne J, Curran S, McAuliffe FM. The influence of a low glycemic index dietary intervention on maternal dietary intake, glycemic index and gestational weight gain during pregnancy: a randomized controlled trial. Nutr J. 2013;12:140.

29. Harrington J, Perry I, Lutomski J, Morgan K, McGee H, Shelley E, Watson D, Barry M. SLÁN 2007: Survey of Lifestyle, Attitudes and Nutrition in Ireland. Dietary Habits of the Irish Population. Dublin: Department of Health a Children; 2008. http://epubs.rcsi.ie/cgi/viewcontent.cgi?article=1005\&amp; context=psycholrep. Accessed 8 May 2015.

30. Gardosi J F. A Customised Weight Centile Calculator. GROW version 6.2.3: Gestation Network. 2007.

31. Horan M, Gibney E, Molloy E, McAuliffe F. Methodologies to assess paediatric adiposity. Ir J Med Sci. 2014;184:53-68.

32. Onis M. WHO child growth standards based on length/height, weight and age. Acta Paediatr. 2006;95:76-85.

33. Bailey D, Perumal N, Yazdanpanah M, Al Mahmud A, Baqui AH, Adeli K, et al. Maternal-fetal-infant dynamics of the C3-epimer of 25-hydroxyvitamin D. Clin Biochem. 2014;47:816-22.

34. Carter GD, Berry JL, Gunter E, Jones G, Jones JC, Makin HLJ, et al. Proficiency testing of 25-Hydroxyvitamin D (25-OHD) assays. J Steroid Biochem Mol Biol. 2010;121:176-9.

35. Aloia JF, Dhaliwal R, Shieh A, Mikhail M, Fazzari M, Ragolia L, et al. Vitamin D supplementation increases calcium absorption without a threshold effect. Am J Clin Nutr. 2013;99:624-31.

36. Zhu K, Prince RL. Calcium and bone. Clin Biochem. 2012;45:936-42.

37. McKenna MJ, Freaney R, Meade A, Muldowney FP. Hypovitaminosis D and elevated serum alkaline phosphatase in elderly Irish people. Am J Clin Nutr. 1985;41:101-9.

38. McKenna MJ. Differences in vitamin D status between countries in young adults and the elderly. Am J Med. 1992;93:69-77.

39. McKenna MJ, Freaney R, Byrne P, McBrinn Y, Murray B, Kelly M, et al. Safety and efficacy of increasing wintertime vitamin $D$ and calcium intake by milk fortification. QJM. 1995;88:895-8.

40. Lonergan R, Kinsella K, Fitzpatrick P, Brady J, Murray B, Dunne C, et al. Multiple sclerosis prevalence in Ireland: relationship to vitamin $D$ status and HLA genotype. J Neurol Neurosurg Psychiatry. 2011;82:317-22.

41. McCarthy RA, McKenna MJ, Oyefeso O, Uduma O, Murray BF, Brady JJ, et al. Vitamin D nutritional status in preterm infants and response to supplementation. Br J Nutr. 2012;110:156-63.

42. Leffelaar ER, Vrijkotte TGM, van Eijsden M. Maternal early pregnancy vitamin D status in relation to fetal and neonatal growth: results of the multi-ethnic Amsterdam born children and their development cohort. Br J Nutr. 2010;104:108-17

43. Morley R, Carlin JB, Pasco JA, Wark JD. Maternal 25-hydroxyvitamin D and parathyroid hormone concentrations and offspring birth size. J Clin Endocrinol Metab. 2006;91:906-12.

44. Sayers A, Tobias JH. Estimated maternal ultraviolet B exposure levels in pregnancy influence skeletal development of the child. J Clin Endocrinol Metab. 2009;94:765-71

45. Van Eijsden M, Snijder MB, Brouwer I, Vrijkotte TGM. Maternal early-pregnancy vitamin $D$ status in relation to linear growth at the age of 5-6 years: results of the ABCD cohort. Eur J Clin Nutr. 2013;67:972-7.

46. Jensen CB, Gamborg M, Raymond K, McGrath J, TIA S, Heitmann BL. Secular trends in seasonal variation in birth weight. Early Hum Dev. 2015;91:361-5.

47. McKenna MJ, Murray BF, O'Keane M, Kilbane MT. Rising trend in vitamin D status from 1993 to 2013: dual concerns for the future. Endocr Connect. 2015. doi:10.1530/EC-15-0037.

\section{Submit your next manuscript to BioMed Central and we will help you at every step:}

- We accept pre-submission inquiries

- Our selector tool helps you to find the most relevant journal

- We provide round the clock customer support

- Convenient online submission

- Thorough peer review

- Inclusion in PubMed and all major indexing services

- Maximum visibility for your research

Submit your manuscript at www.biomedcentral.com/submit
Biomed Central 